Arch Virol (1997) 142: 2499-2514

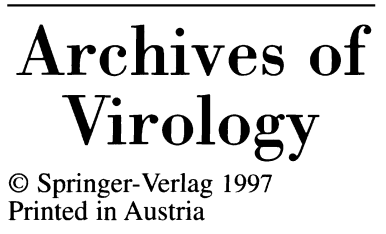

\title{
The $5^{\prime}$-terminal 32 basepairs conserved between genome segments A and B contain a major promoter element of infectious bursal disease virus
}

\author{
M. M. Nagarajan and F. S. B. Kibenge \\ Department of Pathology and Microbiology, Atlantic Veterinary College University \\ of Prince Edward Island, Charlottetown, Canada
}

Accepted June 25, 1997

Summary. The regions of the infectious bursal disease virus (IBDV) genome with regulatory function are not known. In the present study, progressively deleted lengths of the $5^{\prime}$ noncoding region of segment A were constructed in pGL3 vectors having SV40 enhancer or promoter, and a luciferase (LUC) reporter gene. Transient transfections of the constructs made in a promoter-less pGL3-Enhancer vector when transfected in Vero cells and the lysates assayed for LUC expression, allowed the localization of maximal activity to the 32nucleotide stretch (precursor polyprotein ORF positions -131 to -100 ), which is highly conserved at the $5^{\prime}$ end of both genome segments. This fragment, when evaluated in parallel in an enhancer-less pGL3-Promoter vector demonstrated no activity. To determine if this region is recognized by IBDV replicative proteins, we engineered modifications in an enhancer-less pGL3Promoter vector where the terminal 32-bp fragment, the full-length noncoding region, or the noncodig region with the 32-bp fragment deleted was positioned in either the plus-sense or the minus-sense orientation immediately downstream of the SV40 promoter and upstream of the LUC gene. Transfections of these constructs in IBDV-infected and uninfected Vero cells resulted in the endogenous generation of recombinant viral-LUC RNAs containing the $5^{\prime}$ terminal viral RNA sequences in either the plus-sense or the minus-sense orientation. LUC assays of the infected cell lysates showed up-regulated expression of LUC only with constructs containing the 32-bp fragment in the minus-sense orientation. Deletion of this 32-bp fragment abolished such LUC expression. We therefore conclude that the $5^{\prime}$-terminal 32 base pairs of genomic segment A contain a major promoter element in IBDV. In addition, our results show that IBDV replicative proteins recognize and transcribe single-stranded RNA in vivo. 


\section{Introduction}

Infectious bursal disease virus (IBDV) is a member of the genus Avibirnavirus of the family Birnaviridae. Members of this family are characterized by a double-stranded (ds)RNA genome consisting of two segments, A and B, within a non-enveloped single-shelled icosahedral capsid of $60-65 \mathrm{~nm}$ diameter [7]. The genome segments A (approx. $3300 \mathrm{bp}$ ) and B (approx. $2820 \mathrm{bp}$ ) have the putative viral RNA polymerase attached to their $5^{\prime}$ ends through a phosphodiester bond between a guanine residue and a serine [4], forming a genome linked protein, $\operatorname{VPg}[5,24]$. This viral protein, which is encoded by segment B [23], also occurs in virions as a $90 \mathrm{kDa}$ polypeptide (VP1) linked to short stretches of viral RNA [14]. VP1 of IBDV has sequence similarity with known RNA-dependent RNA polymerases of other dsRNA and positive singlestranded (ss) RNA viruses [8]. Segment A encodes five viral proteins from two partially overlapping open reading frames (ORF): VP5 $(16.5-17 \mathrm{kDa})$ from the small ORF $[16,17]$ and a $109-\mathrm{kDa}$ precursor polyprotein from the larger ORF, that is processed into structural proteins VP2 $(40-45 \mathrm{kDa}), \mathrm{VP} 3(32-34 \mathrm{kDa})$ and VP4 $(28-30.5 \mathrm{kDa})[3,12,15,17]$. VP4 is also a viral protease [1]. The function of VP5 is not known.

All dsRNA viruses require the introduction of a virion-associated polymerase complex into the host cell and its subsequent activation to initiate the viral replication cycle. The biochemical processes underlying this activation of transcription have been studied in several dsRNA viruses [13, 21, 33, 34]. In birnaviruses, transcription is initiated when the virus penetrates the host cell without the need for uncoating or degradation of the capsid, and the precusor molecules gain access to the viral polymerase-template complex by passing through the capsid [33]. Recent experiments with infectious pancreatic necrosis virus (IPNV) and IBDV indicate that virion-associated VP1 also catalyzes a guanylylation reaction which serves to prime viral RNA synthesis; apparently, only the plus strands of the two genome segments are synthesized in vitro, which remain base-paired to their minus strand templates [6]. However, the regulatory sequences involved in IBDV RNA synthesis have received no experimental attention.

Most recently, the complete noncoding sequences of the genomic segments $\mathrm{A}$ and $\mathrm{B}$ of IBDV have been determined, showing that both segments are $85.3 \%$ identical in the initial 32 nucleotides at the $5^{\prime}$ end of the plus strands $[18,25]$. In the North American strains of IBDV, the last 5 nucleotides at the $3^{\prime}$ end of the plus strands are also identical between the genome segments [18]. In this investigation, we use transient transfections in Vero cells with a series of pGL3Promoter and -Enhancer vector constructs of progressively deleted lengths of the viral noncoding regions ligated to a luciferase (LUC) reporter gene (Promega) to map the cis-acting regulatory sequences of IBDV. To determine if the $5^{\prime}$ noncoding region is recognized by the IBDV replicative proteins, transient transfections were performed in Vero cells infected with IBDV. For this, modified pGL3-Promoter recombinant constructs were used where the 
terminal 32-bp fragment, the full-length noncoding region, and the noncoding region with the 32-bp fragment deleted were inserted either in plus-sense or minus-sense orientation, flanked upstream by the SV40 promoter and immediately downstream by a LUC reporter gene. Here we demonstrate that the 5'-terminal 32-nucleotide stretch of segment A of IBDV contains a major promoter element for IBDV genome replication and expression.

\section{Materials and methods}

\section{Virus and cells}

IBDV strain $\mathrm{OH}$ of serotype 2 was the source of all cDNA sequences used in this study. Vero cells were used to propagate the virus and for transient plasmid DNA transfections. Vero cell monolayers were grown in Eagle's Minimal Essential Medium (EMEM) supplemented with $10 \%$ fetal bovine serum (FBS) which was reduced to $2 \%$ in the maintenance medium.

\section{Reverse transcriptase-polymerase chain reaction and cDNA cloning}

For first strand cDNA synthesis, purified IBDV dsRNA in 90\% DMSO and IBDV-specific primer SA06 (nt 1520-1539), 5'-TCT CGA TTT GCA TGG GCT AG-3', were used to copy $5^{\prime}$ end plus strand RNA of segment A with the SuperScript II RT (Gibco-BRL) as previously described [18]. The cDNA clone containing the complete $5^{\prime}$ noncoding region (corresponding to precursor polyprotein ORF positions -131 to +12 ) was amplified by PCR with primers SA11 (nt-1-18), 5'-gcg acg cgt GGA TAC GAT CGG TCT GAA-3' ${ }^{\prime}$, and SA12 (nt 126-143), 5'-cta gat ctC AGG TTT GTC ATC GCT GC-3', from the IBDV genome segment A sequence [18], engineered to contain restriction sites $M l u$ I and $B g l$ II (underlined; the non-viral sequences are indicated in lowercase letters), respectively. The resulting PCR product was subcloned into pCRII vector (Invitrogen) according to the manufacturer's protocol to give clone pPIIA containing the insert cDNA of IBDV segment A $5^{\prime}$ terminal region (nt-1-143). The sequence of this clone was verified using the standard dideoxy chain termination method [31].

\section{Plasmid DNA constructions}

Clone pPIIA was the source of the full-length $5^{\prime}$ noncoding region of OH-IBDV genome segment A cDNA. The $M l u$ I- $B G l$ II insert DNA of this clone was subcloned into the $M l u \mathrm{I}$ and $B g l$ II polylinker sites of both pGL3-Promoter GeneLight vector and the pGL3Enhancer GeneLight vector (Promega) immediately upstream of the LUC reporter gene to give clones pPA131 and pEA131, respectively (Fig. 1). The pGL3-Promoter vector contains an SV40 promoter (enhancer-less pGL3-Promoter) while the pGL3-Enhancer vector contains an SV40 enhancer (promoter-less pGL3-Enhancer) and the pGL3-Control vector contains both SV40 promoter and enhancer. All the vectors have the firefly luciferase gene as the genetic reporter (Fig. 1).

Progressive deletions in the $5^{\prime}$ noncoding region of OH-IBDV genome segment A (Fig. 1) were created by digestion of pEA131 with Kpn I (nt 5 in the polylinker region of pGL3 vectors) to produce a $3^{\prime}$ overhang followed by treatment with Exonuclease III [29]. The products were treated with S1 nuclease to remove the single-stranded regions followed by Klenow fill-in and were subsequently self ligated with T4 DNA ligase [29]. Deletion 
A

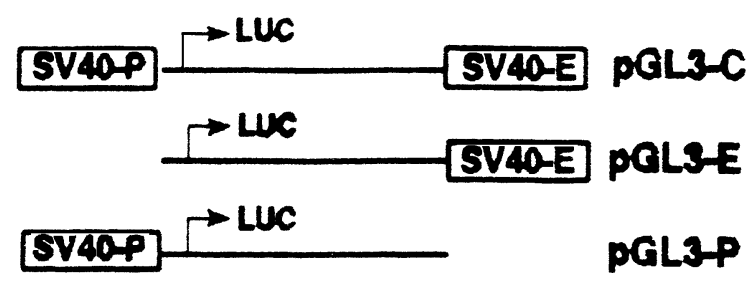

B
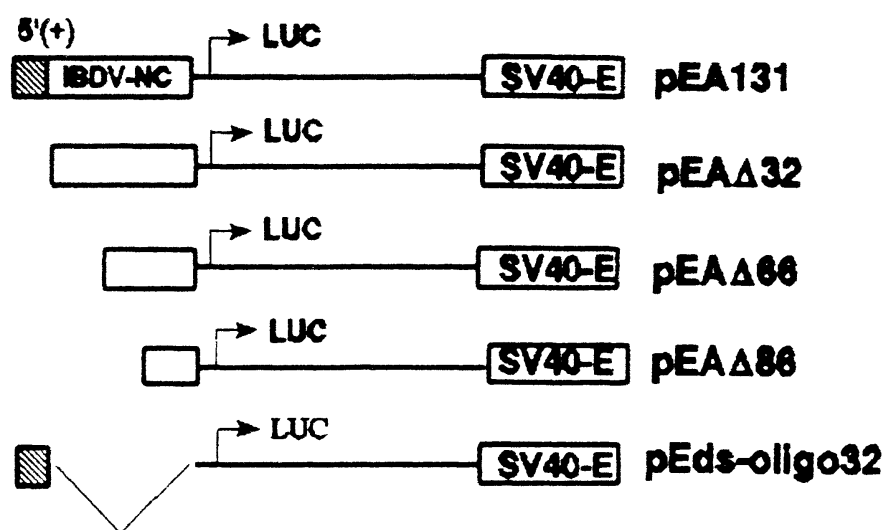

C

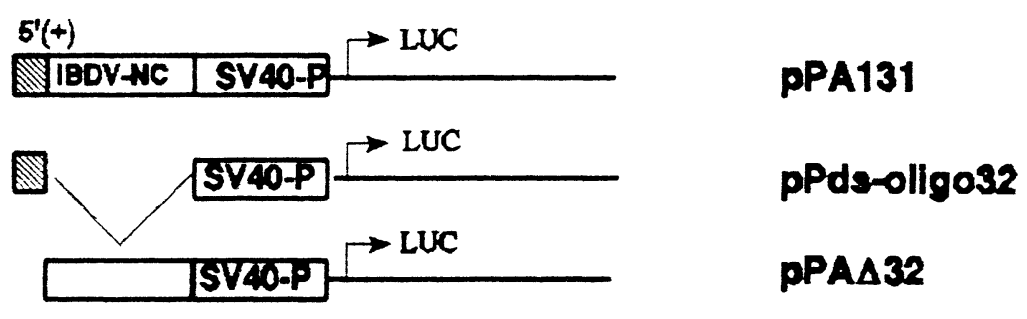

D

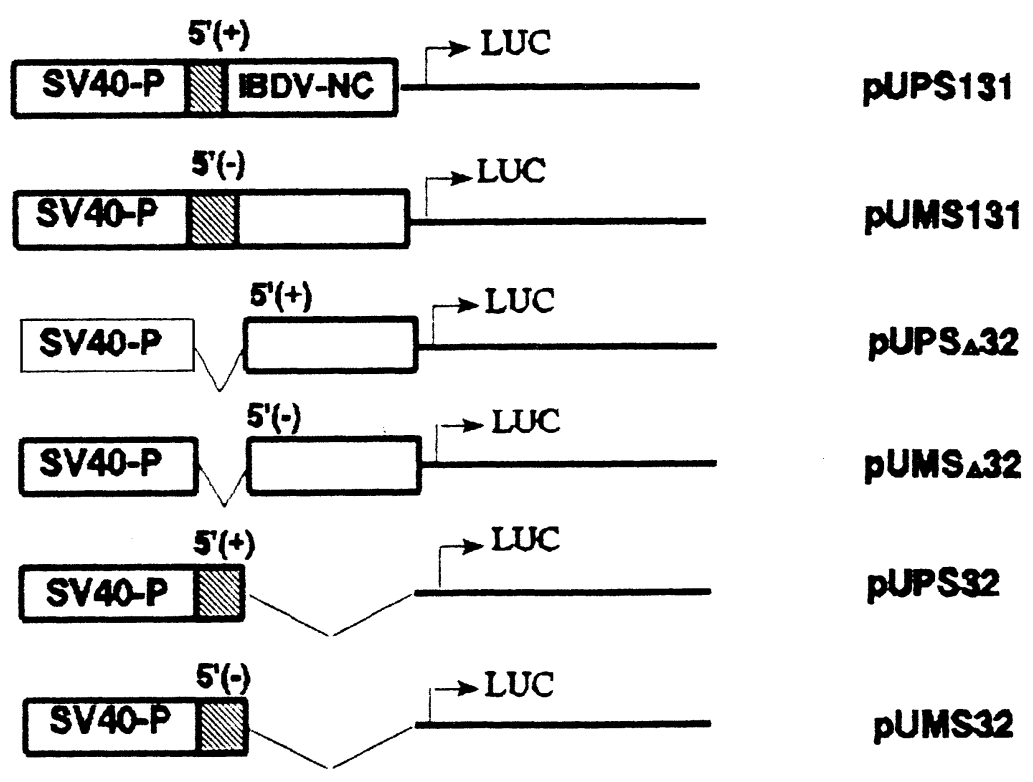


endpoints of the selected plasmid constructs were verified by sequencing using the standard dideoxy chain termination method [31].

To construct pPds-oligo 32 and pEds-oligo 32 clones containing the $5^{\prime}$ end 32-bp cDNA of OH-IBDV genome segment A, an $M l u$ I- $B g l$ II ds DNA fragment containing the initial 32 nucleotides of the $5^{\prime}$ terminal sequence of segment A was commercially synthesized (Bio/Can) and subcloned into the $M l u$ I and $B g l$ II sites of the pGL3-Promoter and pGL3Enhancer vectors, respectively (Fig. 1).

Another set of pGL3-Promoter vector constructs (pUPS32, pUMS32, pUPS131, pUMS131, pUPS $\Delta 32$, and pUMS $\Delta 32$ ) (Fig. 1D) for transfection of uninfected and IBDVinfected Vero cells was engineered from the pGL3-Enhancer recombinant construct (pEA 131) containing the full-length $5^{\prime}$ noncoding region of IBDV by PCR using appropriate sets of primers with the Nco I restriction site, in order to facilitate cloning of the PCR products at the Nco I site of pGL3-Promoter vector. These amplified PCR products containing the terminal $32 \mathrm{bp}$, the full-length noncoding region or the noncoding region with the 32-bp fragment deleted were initially subcloned into pCRII vector and subsequently into the pGL3-Promoter vector at the Nco I site immediately upstream of the LUC reporter gene and downstream of the SV40 promoter (Fig. 1D). Transformants were screened for plussense and minus-sense orientation clones using appropriate primers [30] and the sequences of the selected clones were confirmed using the standard dideoxy chain termination method [31].

\section{Transient transfection of uninfected and IBDV-infected Vero cells}

Both uninfected and IBDV-infected Vero cells were transfected with $1.5 \mu \mathrm{g}$ of plasmid DNA by the Lipofectin method (Gibco-BRL) with some modifications. Briefly, plasmid DNA in $100 \mu$ l of OptiMEM medium (Gibco-BRL) was gently mixed with $10 \mu l$ of

Fig. 1. Plasmid DNA constructs. A pGL3-C denotes the pGL3-Control plasmid with SV40 promoter (SV40-P), luciferase (LUC) reporter gene, and SV40 enhancer (SV40-E); pGL3E denotes pGL3-Enhancer plasmid without SV40 promoter; pGL3-P denotes pGL3Promoter plasmid without SV40 enhancer. B Constructs of pGL3-Enhancer vector containing progressively deleted lengths of the $5^{\prime}$ terminal region of segment A of IBDV (IBDV-5'TR); pEA131 contains the full-length noncoding region (precursor polyprotein ORF positions -131 to +12 ); $\mathrm{pEA} \Delta 32$, pEA $\Delta 66$, and pEA $\Delta 86$ correspond to constructs containing -99 to $+12,-12$, and -45 to +12 nts, respectively, of the $5^{\prime}$ terminal region of segment A of IBDV. pEds-oligo32 contains the initial $32 \mathrm{nts}$ of $5^{\prime}$ terminal region of segment A of IBDV genome ( -131 to $-100 \mathrm{nt})$. C Constructs of pGL3-Promoter vector: pPA131 contains with the full-length non-coding region $(-131$ to $+12 \mathrm{nt})$, pPA $\Delta 32$ contains noncoding region with the terminal 32-bp deleted; pPds-oligo32 contains the initial $32 \mathrm{nts}$ of $5^{\prime}$ terminal region of segment A of IBDV genome ( -131 to $\left.-100 \mathrm{nt}\right)$. Cross-hatched box represents IBDV $32 \mathrm{bp}$ consensus sequence in genome segments $\mathrm{A}$ and B. D Modified pGL3-Promoter vector constructs with the viral cDNA inserted upstream of LUC gene and downstream of SV40 promoter: pUPS131 and pUMS131 contain the fulllength noncoding region of segment A of IBDV in plus-sense and minus-sense orientation, respectively, downstream of the SV40 promoter; pUPS32 and pUMS32 contain the terminal 32-bp fragment in plus-sense and minus-sense orientation, respectively, downstream of the SV40 promoter; pUPS $\Delta 32$ and pUMS $\Delta 32$ contain the noncoding region with the terminal 32-bp fragment deleted, in plus-sense and minus-sense orientation, respectively, downstream of the SV40 promoter 
Lipofectin reagent (Gibco-BRL) diluted in $90 \mu \mathrm{l}$ of OptiMEM and was incubated at room temperature for $15 \mathrm{~min}$. Uninfected Vero cells grown to about $70 \%$ confluence in $35-\mathrm{mm}$ tissue culture dishes, and Vero cells previously infected with OH-IBDV at a multiplicity of infection of 1.0 for $8 \mathrm{~h}$, were washed two times with prewarmed OptiMEM and incubated with the appropriate plasmid DNA-Lipofectin-OptiMEM mixture in triplicates at $37^{\circ} \mathrm{C}$ in a humidified incubator with $5 \% \mathrm{CO}_{2}$ in-air-atmosphere. After $6 \mathrm{~h}$ of incubation, the transfection mixture was replaced with $2 \mathrm{ml}$ of EMEM growth medium.

\section{LUC assays}

Cells were lysed after $48 \mathrm{~h}$ by incubating with $175 \mu \mathrm{l}$ of Cell Lysis reagent (Promega) for $10 \mathrm{~min}$ at room temperature. The LUC assays were carried out at room temperature by mixing $20 \mu \mathrm{l}$ of clarified cell lysate with $100 \mu \mathrm{l}$ of Luciferase Assay reagent (Promega) and monitoring the reaction in a scintillation counter (Packard) for $30 \mathrm{sec}$ according to the manufacturers' protocols. For each experiment, serial dilutions of standard luciferase (Sigma) were carried out to confirm the linearity of the assay. Experimental controls included the pGL3-Control, -Promoter, and -Enhancer vector DNA transfected, mock transfected, uninfected and IBDV-infected Vero cell lysates. The plasmid construct yielding the maximum LUC activity level in an experiment was considered to have $100 \%$ activity, and all other constructs in the experimental group were represented arbitrarily relative to the construct with maximum activity.

\section{Results}

\section{Localization of promoter strength in the $5^{\prime}$ noncoding region of IBDV segment $A$}

Transfections of Vero cells with a series of promoter-less pGL3-Enhancer vector constructs containing a LUC reporter gene encompassing progressively deleted lengths of the $5^{\prime}$ noncoding sequences of segment A (Fig. 1B) were used to map promoter sequences in the IBDV genome. Vero cells transfected with the recombinant pEA131 which contained the full-length $5^{\prime}$ noncoding sequence had $7.5 \%$ LUC activity relative to that of the pGL3-Control vector whereas Vero cells transfected with constructs with the first 32, 66, and $86 \mathrm{bp}$ deleted [pEA $\Delta 32$, pEA $\Delta 66$, and pEA $\delta 86$, respectively (Fig. 1B)], had negligible LUC activity (Fig. 2A). These results suggested that the terminal 32 nucleotides at the $5^{\prime}$ end of genome segment A may contain viral regulatory sequences.

To further determine the regulatory activity of the 32-bp fragment of IBDV genome segment $\mathrm{A}$, a construct containing only the first $32 \mathrm{bp}$ (pEds-oligo32) was prepared in a promoter-less pGL3 vector (Fig. 1B) and transfected in Vero cells. The LUC activity of pEds-oligo 32 was $54.8 \%$ relative to that of the pGL3-Control vector, and represented a $7.8 \%$-fold increase over that of the pEA131 construct containing the full-length $5^{\prime}$ noncoding region. Figure 2B shows the LUC activity of constructs pEA131, pEA $\Delta 32$, pEA $\Delta 66$, and the pGL3-Control vector relative to pEds-oligo32. These data indicate the presence of a strong promoter element in the first $32 \mathrm{bp}$ of the $5^{\prime}$ terminal noncoding region of IBDV segment A. 


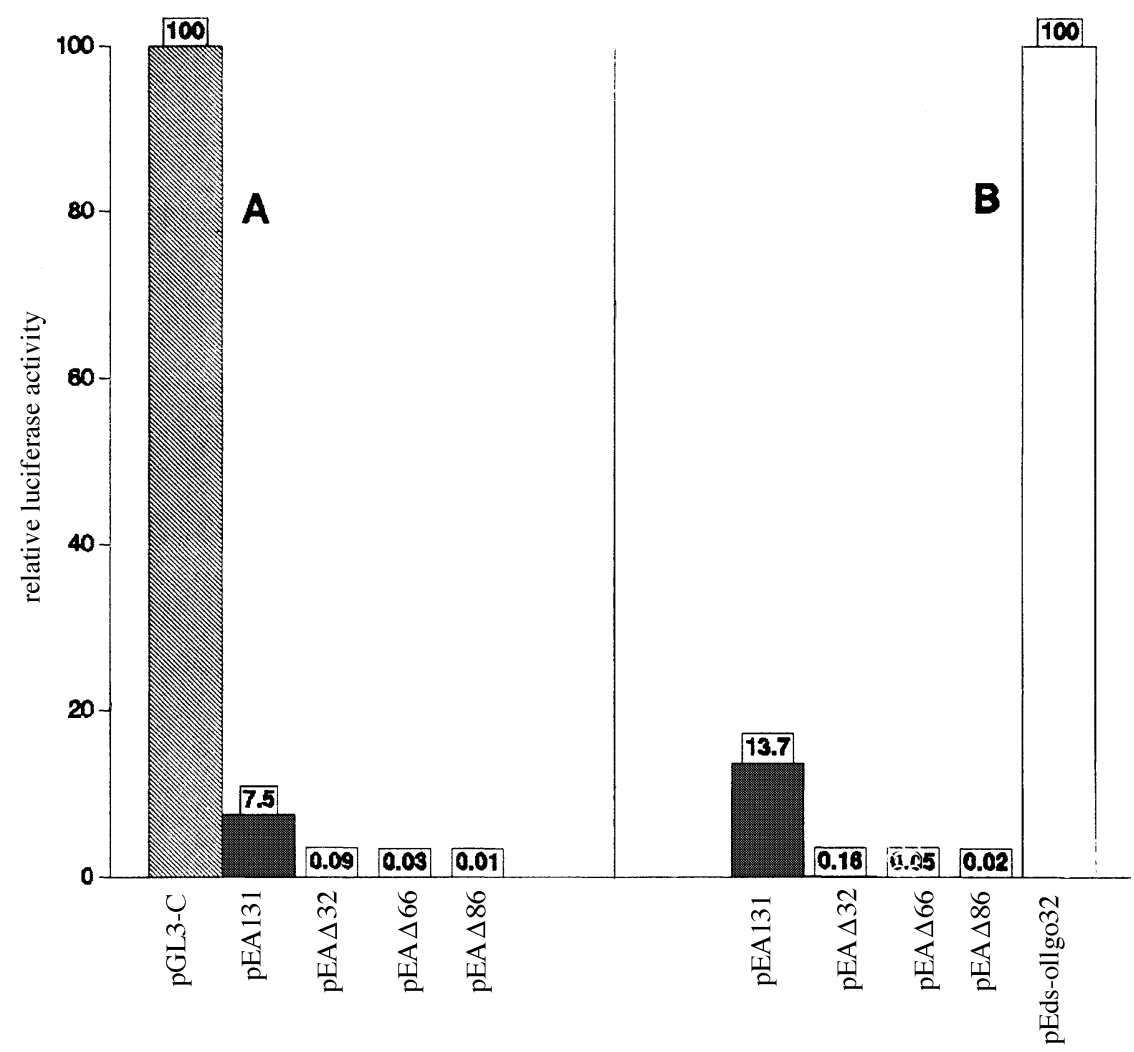

Fig. 2. Promoter strength of the $5^{\prime}$ noncoding region of segment A of IBDV. The luciferase levels are arbitrarily relative to that of pGL3-Control vector (pGL3-C). A LUC activity when pEA131, pEA $\Delta 32$, pEA $\Delta 66$, and pEA $\Delta 86$ were initially examined in parallel to that of pGL3-Control vector, and represent the results of at least three independent experiments. Standard error of the mean was less than $11 \%$ in all cases. B Relative LUC levels when the same constructs were tested in parallel with pEds-oligo32 and pGL3-Control vector (pGL3-C). The luciferase activity levels are represented relative to that of the pEds-oligo32 construct

\section{Analysis of the 32-bp $5^{\prime}$ terminal sequence of IBDV segment A} for enhancer activity

To rule out enhancer activities in this region, we also made enhancer-less pGL3 constructs containing the full-length noncoding region (pPA131), the first $32 \mathrm{bp}$ of the $5^{\prime}$ end of segment A (pPds-oligo32) and the $5^{\prime}$ noncoding region with the first $32 \mathrm{bp}$ deleted (pPA $\Delta 32$ ) (Fig. 1C). These constructs were transfected in Vero cells in parallel with the pGL3-Control vector. As shown in Fig. 3, pPdsoligo32 had no LUC activity and therefore no ehnancer activity whereas pPA131 and pPA $\triangle 32$ exhibited $27 \%$ and $8.2 \%$ LUC activity, respectively, compared to the pGL3-Control vector. 


\section{The first 32-bp 5'-terminal sequence of segment $A$ is a major IBDV promoter region}

To demonstrate the biological importance of this 32-bp 5'-terminal sequence for IBDV genome replication and expression, transfection assays were carried out in IBDV-infected and uninfected Vero cells with a different set of pGL3promoter vector constructs that were engineered to contain either the plus-sense or the minus-sense full-length noncoding region (pUPS131, pUMS131) or with the first $32 \mathrm{bp}$ deleted (pUPS $\Delta 32$, pUMS $\Delta 32$ ) or containing only the first $32 \mathrm{bp}$ of the $5^{\prime}$ noncoding region (pUPS32, pUMS32) downstream of the SV40 promoter (Fig. 1D). These constructs, upon transfection in IBDV-infected Vero cells, allowed the generation of endogenous plus-sense LUC RNAs by cellular RNA polymerase from the SV40 promoter. This RNA contained immediately upstream of the LUC initiation codon the minus-sense or the plus-sense respective viral RNAs. Figure 4 shows nearly 3 and 7-fold up-regulated LUC expression levels by the minus-sense full-length (pUMS131) and terminal 32bp fragment (pUMS32) constructs, respectively, over those of the corresponding constructs with plus-sense orientation (pUPS131 and pUPS32). Such amplified expression levels exhibited by pUMS131 and pUMS32 constructs were in marked contrast to those of the two constructs where the terminal 32-bp fragment was deleted (pUPS $\Delta 32$ and pUMS $\Delta 32$ ) which showed no increase in

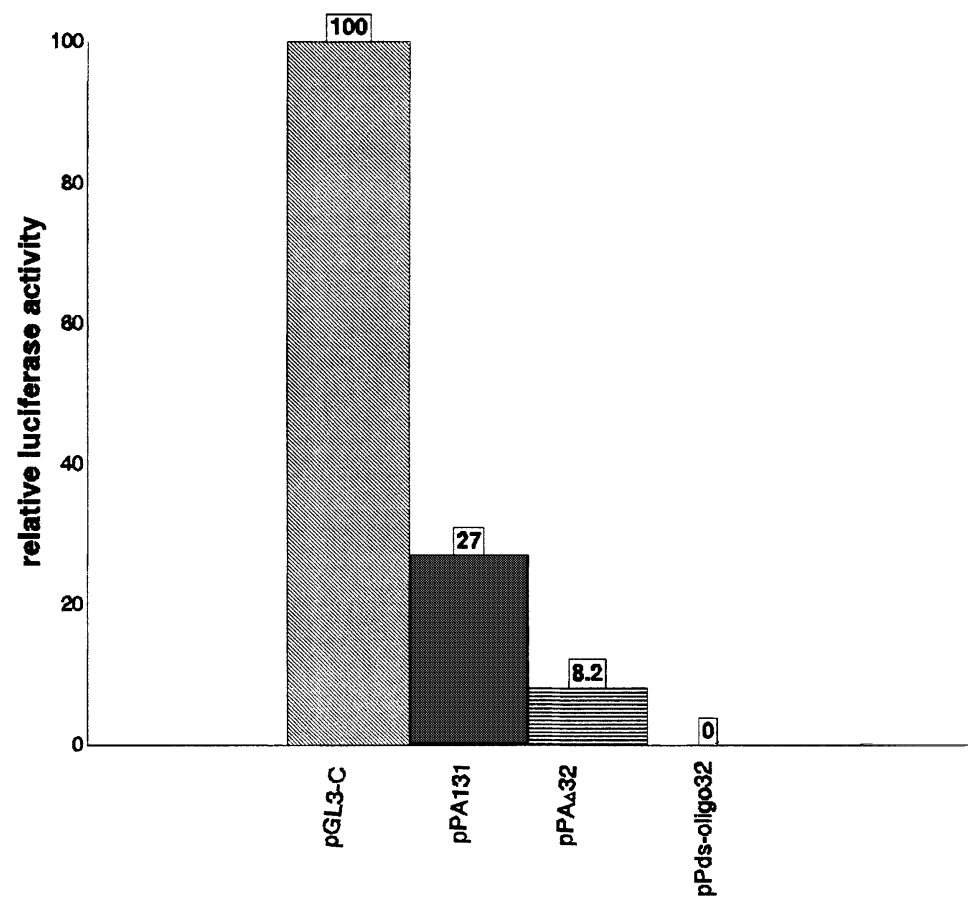

Fig. 3. Enhancer strength of the $5^{\prime}$ noncoding region of segment A of IBDV. The luciferase activity levels of pGL3-Promoter constructs pPA131, pPA $\Delta 32$, and pPds-oligo32 are represented here arbitrarily relative to that of pGL3-Control vector 
LUC expression in IBDV-infected cells. These data showed that IBDV replicative proteins in the IBDV-infected Vero cells recognized the regulatory regions in the $5^{\prime}$ noncoding region resulting in modulation of the LUC expression. In uninfected cells, slightly increased LUC activity was detected only with constructs containing the full-length noncoding region (pUPS131 and pUMS131) and the noncoding region with the 32-bp fragment deleted (pUPS $\Delta 32$ and pUMS $\Delta 32$ ), which was attributed to the presence of enhancer-like sequences in the noncoding region. Constructs containing only the terminal 32-bp fragment (pUPS32 and pUMS32) showed no such increase in LUC activity when transfected in uninfected cells, in agreement with data from the transfection experiments with the pPds-oligo32 construct (Fig. 3). The transfected pGL3-Promoter (pGL3-P) vector in Fig. 4 shows slightly higher LUC activity over some of the other constructs used in infected and uninfected cells because the viral cDNA inserts were ligated at the Nco I site immediately upstream of the LUC initiation codon instead of the polylinker site as in pGL3Promoter GeneLight vector (Promega). This resulted in relatively lower translation efficiency for all the modified constructs (Fig. 1D), due to displacement of the Kozak consensus sequence $5^{\prime}$ of the LUC gene of pGL3-Promoter GeneLight vector (Promega).

\section{Discussion}

The $5^{\prime}$ terminal sequence of the plus strand RNA in both genome segments consists of a 32-base consensus sequence that is identical in all six IBDV strains examined to date whereas the $3^{\prime}$ end is variable except for the last 5 bases which are similar [18]. Transcription of the birnavirus genome is by a semiconservative strand displacement mechanism, with only the plus strand RNAs of the two genome segments synthesized in vitro [6]. Plus-sense RNA transcripts synthesized in vivo are either sequestered by ribosomes for translation, or are packaged into empty procapsids, where they serve as templates for minus strand synthesis similar to what has been reported for other dsRNA viruses [10, 27]. We present here for the first time, data that demonstrates that the initial 32-bp stretch of sequence at the $5^{\prime}$ end of genome segment A (precursor polyprotein ORF positions -131 to -100 ) is a major promoter element of IBDV.

In the first set of transfection experiments in uninfected Vero cells, with progressively deleted constructs of the $5^{\prime}$ noncoding region of segment $\mathrm{A}$, only the full-length construct (pEA131) and the construct with the first 32-bp fragment (pEds-oligo32) had significant LUC activity when examined for promoter strength. The full-length construct (pPA131) and the construct of the noncoding region with the terminal 32-bp fragment deleted (pPA $\Delta 32$ ), but not the construct with the terminal 32-bp fragment (pPds-oligo32), also showed LUC activity when tested for enhancer activity (Fig. 3). The promoter and enhancer activities of the these constructs in uninfected Vero cells were unexpected as there is no report of sequence identity between IBDV VP1 and DNA-dependent RNA polymerases. However, since Vero cells are permissive to 
IBDV replication, it is possible that the viral cDNA inserts in the constructs were recognized by cellular transcription factors thereby modulating LUC expression in uninfected Vero cells.

To further determine whether the apparent promoter activity of the terminal 32-bp fragment could be recognized by IBDV replicative proteins, selected regions of IBDV segment A terminal RNA sequences were generated endogenously in vivo. For this, transiently transfected modified pGL3-Promoter constructs were made in which in vivo transcription initiated by cellular RNA polymerase II recognizing the SV40 promoter positioned upstream of the viral terminal cDNA sequences that were flanked downstream by the luciferase coding region (Fig. 1D) would generate recombinant viral-LUC RNAs when transfected in Vero cells. This strategy is analogous to that previously used in the mutational analysis of influenza virus promoter elements in vivo [26], where cellular RNA polymerase I was used for in vivo transcription of transfected constructs containing recombinant viral terminal cDNA sequences with CAT reporter gene. As shown in Fig. 4, virtually all the up-regulated LUC activity in infected cells could be attributed to the endogenous generation of viral minussense RNA of the terminal $32 \mathrm{bp}$ upstream of the LUC AUG codon resulting from the transcription by cellular RNA polymerase II recognizing the SV40

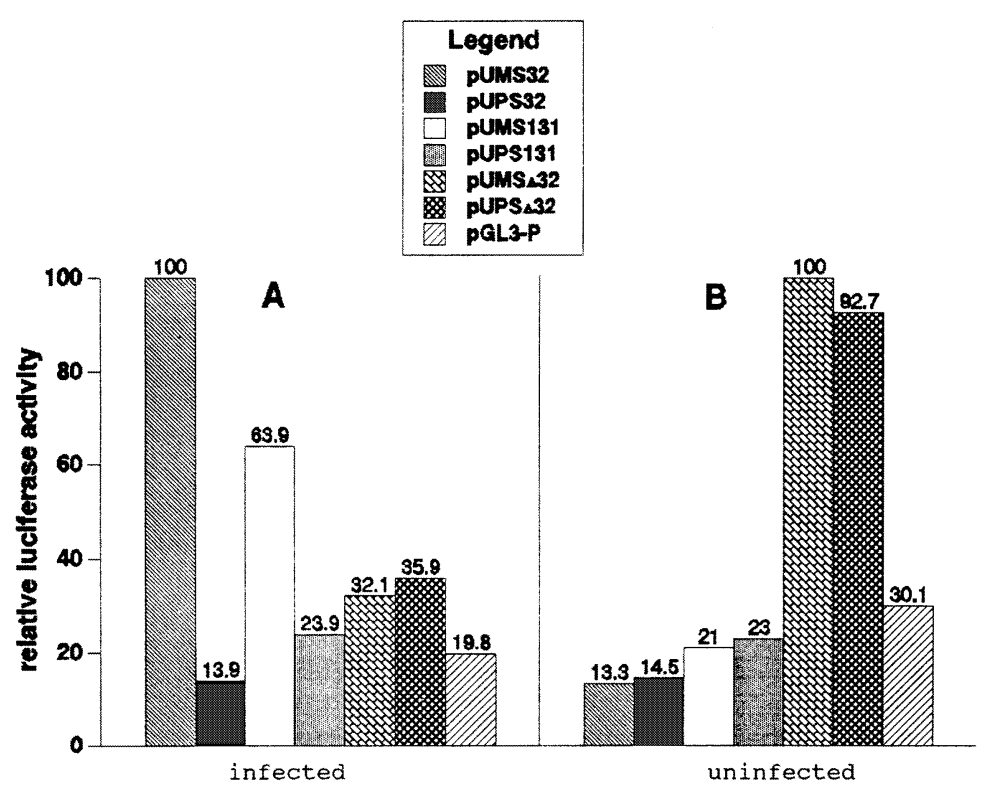

Fig. 4. Comparison of the promoter strength of the $5^{\prime}$ noncoding region of segment $\mathrm{A}$ of IBDV in virus-infected and uninfected Vero cells. The values represent the results of at least three independent experiments in which the constructs pUMS32, pUPS32, pUMS131, pUPS131, pUMS $\Delta 32$ and pUPS $\Delta 32$ were tested in parallel with that of pGL3-Promoter vector. The values of the constructs in $\mathbf{A}$ are represented arbitrarily relative to that of pUMS32 and those in $\mathbf{B}$, relative to that of pUMS $\Delta 32$. The standard error of the mean was 


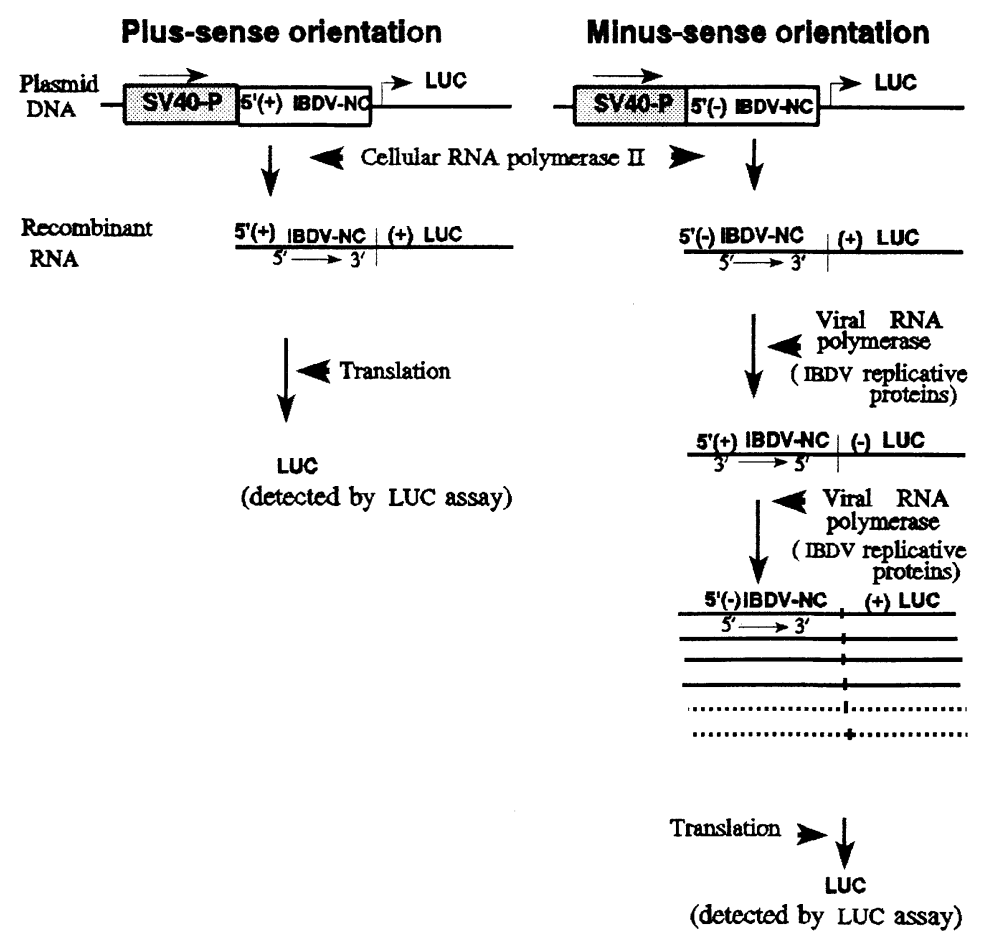

Fig. 5. Schematic diagram of the in vivo transcription of the recombinant viral-LUC DNA constructs. The plasmid DNA with $5^{\prime}(+)$ IBDV-NC represents constructs pUPS131 and pUPS32 with the plus-sense orientation $5^{\prime} \rightarrow 3^{\prime}$ of the noncoding region of segment A of IBDV downstream of the SV40 promoter. The plasmid DNA with $5^{\prime}(-)$ IBDV-NC represents constructs pUMS131 and pUMS32 with the minus-sense orientation $5^{\prime} \rightarrow 3^{\prime}$ of the noncoding region of segment A of IBDV downstream of the SV40 promoter. For the recombinant RNA, $5^{\prime}(+)$ IBDV-NC and $5^{\prime}(-)$ IBDV-NC denote the plus strand RNA and the minus strand RNA, respectively; the $5^{\prime} \rightarrow 3^{\prime}$ or $3^{\prime} \rightarrow 5^{\prime}$ direction is also indicated below each RNA strand

promoter in constructs pUMS32 and pUMS131 (Fig. 1D). Like other viral RNAs with specific cis-acting signals that serve to promote specific viral RNA synthesis [28], the presence of the conserved 32-nucleotide stretch recognized by IBDV replicative proteins would preclude cellular mRNA from entering into the RNA replication of the virus.

Figure 5 depicts the possible sequence of transcription events in the upregulation of LUC expression in IBDV-infected Vero cells. Upon transfection, plasmid DNA constructs were transcribed by cellular RNA polymerase II recognizing the SV40 promoter. The resulting transcripts were either translated into LUC or served as templates for transcription by the viral RNA polymerase provided in trans in IBDV-infected Vero cells. Since transfections with pUMS32 and pUMS131 constructs alone resulted in up-regulated LUC expression (Fig. 4), only the cellular RNA polymerase II transcripts containing 
the $5^{\prime}$-terminal viral RNA sequence in the minus-sense orientation were initially recognized by the viral RNA polymerase. It also follows that the viral RNA polymerase was able to utilize ssRNA as template for RNA synthesis, and that upon recognition of the promoter sequence in the terminal viral RNA, transcription could proceed in either direction. Probably $5^{\prime}-3^{\prime}$ interactions of viral RNA strands are important in determining the direction of transcription. Because of amplification in LUC expression in pUMS32 and pUMS131, the LUC transcripts used for translation must have been at least the second generation transcripts of the viral RNA polymerase. This implies that the viral RNA polymerase was also able to use its own plus-sense transcripts as templates for transcription. Since the plus-sense transcripts of cellular RNA polymerase II (in $5^{\prime}-3^{\prime}$ orientation) were apparently not used as templates for transcription, it is possible that the plus-sense transcripts of the viral RNA polymerase (in $3^{\prime}-5^{\prime}$ orientation) were somehow modified. It is not known whether the difference in orientation of the plus-sense transcripts was sufficient to allow the viral RNA polymerase to utilize its own plus-sense transcripts as templates for RNA synthesis. However, the results clearly indicate that while the viral terminal sequences of both negative and positive polarities served as templates for transcription by the viral RNA polymerase, the plus-sense templates were transcribed only if they were transcription products of the viral RNA polymerase (Fig. 5). This would account for the differences in LUC expression in IBDV-infected cells transfected with constructs in minus-sense and plus-sense orientations (Fig. 4).

It is possible that the regions analyzed for regulatory functions in the present study might have exerted differential translation efficiencies on the in vivo generated recombinant viral-LUC RNA transcripts. However, the absence of higher levels of expression in the constructs lacking the viral terminal 32-bp fragment (pUPS $\Delta 32$ and pUMS $\Delta 32$ ) in transfected infected cells in contrast to the strong stimulation by constructs with the 32-bp fragment (pUMS32 and pUMS131) (Fig. 4) indicates that such differential translation efficiencies were not a factor in the up-regulation of LUC expression, and that IBDV-specific amplification indeed took place rendering endogenously generated terminal viral RNA sequences from these constructs biologically active. Moreover, the LUC assays of the uninfected cells transfected with the same pGL3-Promoter recombinant constructs (Fig. 4) when ran in close parallel with those of the initial transfections (Fig. 3) ruled out any enhancer activity in the viral terminal 32-bp fragment. Additional evidence consistent with our interpretation that the 5'-terminal 32-nucleotide stretch of genome segment A contains a major promoter region of IBDV are: (i) this minimal region is highly conserved between the two genome segments [18], suggesting it has functional significance common to the two segments; (ii) this region had maximal promoter strength with no detectable enhancer activity, of the five constructs containing viral noncoding sequences transfected in uninfected cells, and the six promoter vector constructs transfected in IBDV-infected and uninfected cells; and (iii) this sequence at the $3^{\prime}$ end of the minus strands for both genome 


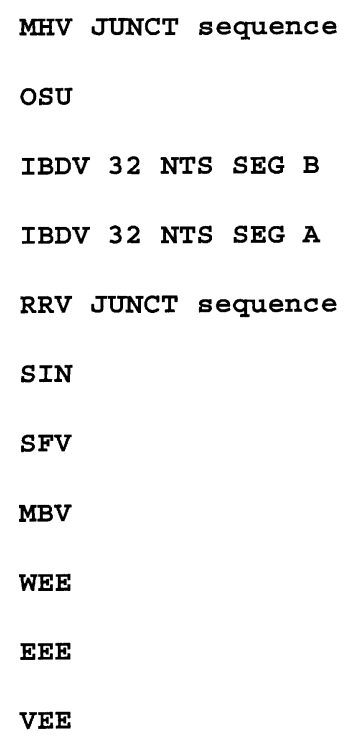

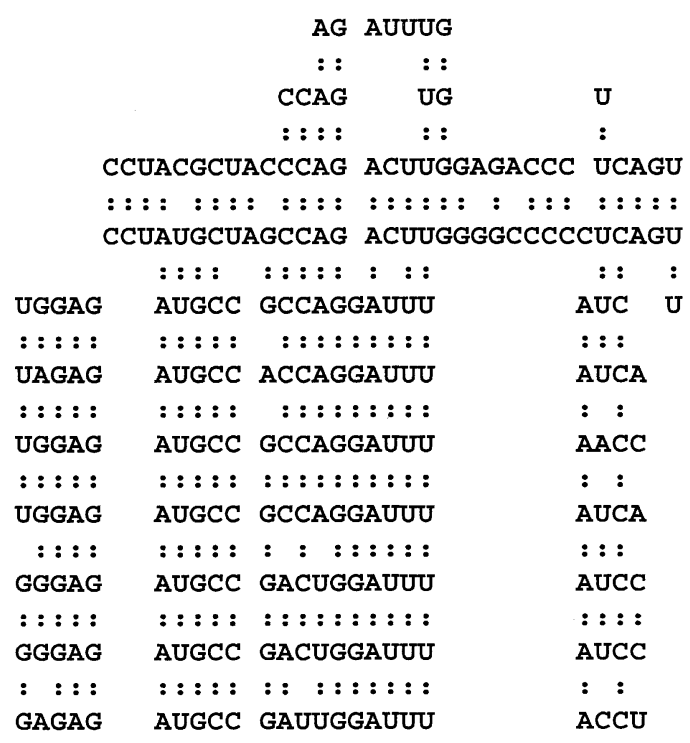

Fig. 6. Alignment of the 32-nucleotide stretch at the $3^{\prime}$ end of the minus strands of genome segments $\mathrm{A}$ and $\mathrm{B}$ of IBDV with minus strand sequences at initiation sites of some alphaviruses [19]; $R R V$ Ross river virus; SIN Sindbis virus; $S F V$ Semliki forest virus; $M B V$ Middleburg virus; WEE Western equine encephalitis virus; EEE Eastern equine encephalitis virus; $V E E$ Venezuelan equine encephalitis virus; polymerase recognition core in rotavirus gene $8 \mathrm{mRNA}$ [28]; and consensus intergenic sequences of mouse hepatitis virus $(M H V)$ [32]. Gaps are introduced to align the sequences. Colons denote sequence identity between viruses and between genome segments A and B of IBDV

segments $\mathrm{A}$ and $\mathrm{B}$ has considerable sequence identity with sequences at the alphavirus initiation site for subgenomic RNA synthesis [20], the polymerase recognition core of rotavirus gene 8 mRNA [28] and the unique consensus intergenic sequences of mouse hepatitis virus [21, 32] (Fig. 6), which have been suggested to play an essential role in transcription. Any nucleotide differences between IBDV segments A and B within the 32-bp conserved region (Fig. 6) may represent positions of lesser importance of recognition by the viral RNA polymerase. By the same token, sequence differences between viruses in the promoter sequences aligned in Fig. 6 may be a reflection of differences in specificities of the various viral RNA polymerases.

The mode of IBDV genome expression is similar to that of dsRNA bacteriophage $\phi 6$ but different from that of other animal dsRNA viruses such as rotavirus and reovirus. Birnaviruses [6] and bacteriophage $\phi 6[9,35,36]$ show a semiconservative strand displacement transcription mechanism in contrast to the conservative mechanism exhibited by rotatirus [11, 27] and reovirus [2, 19]. Birnaviruses are also unique among dsRNA viruses studied so far in having a VPg attached to the $5^{\prime}$ end of both genome segments, circularizing them [24]. Furthermore, they are the only dsRNA viruses which show in vitro viral protein- 
primed RNA synthesis [6]. The regulatory region for genome expression identified on the viral $5^{\prime}$-terminal noncoding region in the present study is likely to play a major role in the virus replication cycle. However, our results do not rule out the possibility that other regulatory sequences could be located at the $3^{\prime}$-terminus of the genome segments as well. The availability of an in vivo assay for viral regulatory regions provides a tool with which to further study these (and other) interactions in birnavirus replication.

\section{Acknowledgements}

The authors are grateful for the technical assistance of Patricia McKenna. This work was supported by research grants from the Natural Sciences and Engineering Research Council of Canada and by the Atlantic Veterinary College Research Fund.

\section{References}

1. Azad AA, Jagadish MN, Brown MA, Hudson PJ (1987) Deletion mapping and expression in Escherichia coli of the large genomic segment of a birnavirus. Virology 143: 35-44

2. Banerjee AK, Shatkin AJ (1970) Transcription in vitro by reovirus associated RNA dependent polymerase. J Virol 6: 1-11

3. Böttcher B, Kiseler NA, Stel'Mashchuk VY, Perevozchikova NA, Borisov AV, Crowther RA (1997) Three-dimensional structure of infectious bursal disease virus determined by electron cryomicroscopy. J Virol 71: 325-330

4. Calvert JG, Nagy É, Soler M, Dobos P (1991) Characterization of the VPg-dsRNA linkage of infectious pancreatic necrosis virus. J Gen Virol 72: 2563-2 567

5. Dobos P (1993) In vitro guanylylation of infectious pancreatic necrosis virus polypeptide. Virology 193: 403-413

6. Dobos P (1995) Protein primed RNA synthesis in vitro by the virion-associated RNA polymerase of infectious pancreatic necrosis virus. J Virol 32: 593-605

7. Dobos P, Berthiaume L, Leong JA, Kibenge FSB, Müller, H, Nicholson BL (1995) Birnaviridae In: Francki RIB, Fauquet CM, Knudson DL, Brown F (eds) Classification and Nomenclature of Viruses. Sixth Report of the International Committee on Taxonomy of Viruses. Springer, Wien New York, pp 240-244 (Arch Virol [suppl]2)

8. Duncan R, Mason, CL, Nagy È, Leong J-A, Dobos P (1991) Sequence analysis of IPNV genome segment B and its encoded VP1 protein. A putative RNA polymerase activity lacking the Gly-Asp-Asp motif. Virology 181: 541-552

9. Emori Y, Iba H, Okada Y (1980) Semi-conservative transcription of double-stranded RNA catalyzed by bacteriophage $\phi 6$ RNA polymerase. J Biochem 88: 1569-1 575

10. Frilander M, Gottlieb P, Strassman J, Bamford DH, Mindich L (1992) Dependence of minus-strand synthesis on complete genomic packaging in the double-stranded RNA bacteriophage $\phi 6$. J Virol 66: 5013-5017

11. Gorziglia MI, Collins PL (1992) Intracellular amplification and expression of a synthetic analog of rotavirus genomic RNA bearing a foreign marker gene. Mapping cis-acting nucleotides in the $3^{\prime}$-noncoding region. Proc Natl Acad Sci USA 89: $5784-$ 5788

12. Hudson PJ, McKern NM, Power PE, Azad AA (1986) Genomic structure of the large RNA segment of infectious bursal disease virus. Nucleic Acids Res 14: 5 001-5012

13. Huismans H, Van Dijk AA, Els HJ (1987) Uncoating of parental bluetongue virus to core and subcore particles in infected L cells. Virology 167: 180-188 
14. Kibenge FSB, Dhama VJ (1997) Evidence that virion-associated VP1 of avibirnaviruses contains viral RNA sequences. Arch Virol 142: 1227-1 236

15. Kibenge FSB, Dhillon AS, Russell RG (1988) Biochemistry and immunology of infectious bursal disease virus. J Gen Virol 69: 1757-1775

16. Kibenge FSB, Jackwood DS, Mercado CC (1990) Nucleotide sequence of genome segment A of infectious bursal disease virus. J Gen Virol 71: 569-577

17. Kibenge FSB, McKenna PK, Dybing JK (1991) Genomic cloning and analysis of the large RNA segment (segment A ) of a naturally avirulent serotype 2 infectious bursal disease virus. Virology 184: 437-440

18. Kibenge FSB, Nagarajan MM, Qian B (1996) Determination of $5^{\prime}$ and $3^{\prime}$ terminal noncoding regions of the bi-segmented genome of the avibirnavirus infectious bursal disease virus. Arch Virol 141: 1133-1 141

19. Klett GAH, Schonberg M, Christman J, Levin DH, Silverstein SC (1971) Mechanism of reovirus double-stranded RNA synthesis in vivo and in vitro. J Virol 8: 684-689

20. Levis R, Schlesinger S, Huang HV (1990) Promoter for Sindbis virus-RNA-dependent subgenomic RNA transcription. J Virol 64: 1726-1733

21. Ludert JE, Michelangeli F, Gil F, Liprandi F, Esparza J (1987) Penetration and uncoating of rotaviruses in cultured cells. Intervirology 27: 95-101

22. Makino S, Joo M, Makino JK (1991) A system for study of coronavirus mRNA synthesis: a regulated, expressed subgenomic defective interfering RNA results from intergenic site insertion. J Virol 65: 6031-6041

23. Morgan MM, MaCreadie IG, Harley VR, Hudson PJ, Azad AA (1988) Sequence of a small double-stranded RNA genome segment of infectious bursal disease virus and its deduced 90-kDa product. Virology 163: 240-242

24. Müller H, Nitschke R (1987) The two segments of infectious bursal disease virus genome are circularized by a 90,000-Da protein. Virology 159: 174-177

25. Mundt E, Müller H (1995) Complete nucleotide sequences of $5^{\prime}$ and $3^{\prime}$-noncoding regions of both genome segments of different strains of infectious bursal disease virus. Virology 209: 10-18

26. Neumann G, Hobom G (1995) Mutational analysis of influenza promoter elements in vivo. J Gen Virol 76: 1709-1719

27. Patton JT (1993) Rotavirus replication. Curr Topics Microbiol Immunol 185: 93-113

28. Patton JT (1995) Structure and function of the rotavirus RNA-binding proteins. J Gen Virol 76: 2633-2644

29. Sambrook J, Fritsch EF, Maniatis T (1989) Molecular cloning: A laboratory manual, 2nd ed. Cold Spring Harbor Laboratory Press, New York

30. Sandhu GS, Precup W, Kline B (1989) Rapid one-step characterization of recombinant vectors by direct analysis of transformed Escherichia coli colonies. BioTechniques 7: 689-690

31. Sanger F, Nicklen S, Coulsen AR (1977) DNA sequencing with chain-terminating inhibitors. Proc Natl Acad Sci USA 74: 5463-5 467

32. Shieh C, Soe LH, Maniko S, Chang M, Stohlman SA, Lai MMC (1987) The $5^{\prime}$-end sequence of the murine coronavirus genome: implications for multiple fusion sites in leader-primed transcription. Virology 156: 321-330

33. Spies U, Müller H, Becht H (1987) Properties of RNA polymerase activity associated with infectious bursal disease virus and characterization of its reaction products. Virus Res 8: $127-140$

34. Stürzenbecker LJ, Nibert M, Furlong D, Fields BN (1987) Intracellular digestion of reovirus particles requires a low $\mathrm{pH}$ and is an essential step in the viral infectious cycle. J Virol 61: 2351-2 361 
2514 M. M. Nagarajan and F. S. B. Kibenge: IBDV major promoter element

35. Usala SJ, Brownstein BH, Haselkorn R (1980) Displacement of parental RNA strands during in vitro transcription by bacteriophage $\phi 6$ nucleocapsids. Cell 19: 855-862

36. Van Etten JL, Burbank DE, Cuppels DA, Lane LC, Vidaver AK (1980) Semiconservative synthesis of single-stranded RNA by bacteriophage $\phi 6$ RNA polymerase. J Virol 33: 769-773

Authors' address: Dr. F. S. B. Kibenge, Department of Pathology and Microbiology, Atlantic Veterinary College, University of Prince Edward Island, 550 University Avenue, Charlottetown, P.E.I., C1A 4P3, Canada.

Received May 14, 1997 